\section{RISK FACTORS ASSOCIATED WITH ANEMIA AND NUTRITIONAL STATUS IN INFANTS}

doi:10.1136/archdischild-2012-302724.0770

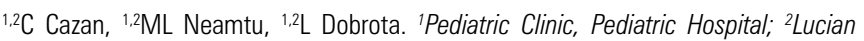
Blaga University of Sibiu, Sibiu, Romania

Background Growth assessment is an integral part of infants health. An understanding of anemia risk factors is essential to identify the groups that are more vulnerable.

The Aim of the study was to identify the risk factors for malnutrition in the vulnerable age group of 6 to 12 months and to evaluate possible related risk factors with anemia.

Methods The cross-sectional study which evaluated 206 infants aged between 6-12 months who attended the Pediatric Clinic during the years 2010-2011 for inadequate growth. Mothers were interviewed to collect informations regarding socioeconomic status and nutrition practices. Nutritional status was evaluated by anthropometric measurements using growth charts. Anemia was diagnosed if hemoglobin was $\leq 11 \mathrm{~g} / \mathrm{dL}$.

Results Mild and moderate anemia was characterised by hemoglobin levels below 11.0 and $9.5 \mathrm{~g} / \mathrm{dL}$. Rates for mild and moderate anemia were $38.6 \%$ and $11.9 \%$. The highest anemia prevalence was found at 6 to 8 months of age. The risk factors for anemia were: urban residence $(p=0.004)$, fever in the past 5-7 days $(p<0.001)$ and age at $6-8$ months $(p=0.024)$, socioeconomic level and nutrition practices. Infants who were exclusively breastfed for 6 months showed lower prevalence of anemia compared to their mixed feeding. According to weight and length for-age, $49 \%$ of the infants were at $25^{\text {th }}, 32 \%$ at $10^{\text {th }}, 9.2 \%$ at $5^{\text {th }}$ and there was a significant correlation between the duration of breastfeeding and nutritional status.

Conclusions Strategies to control infant anemia should include health promotion and nutritional education for families from all socioeconomic levels.

\section{HIGH INCIDENCE OF IRON DEFICIENCY IN YOUNG CHILDREN WITH CYSTIC FIBROSIS}

doi:10.1136/archdischild-2012-302724.0771

L Uijterschout, J Vloemans, M Nuysink, DM Hendriks, F Brus. Juliana Children's Hospital/HAGAZiekenhuis, The Hague, The Netherlands

Background Iron deficiency (ID) is common in patients with cystic fibrosis (CF). In adult CF patients ID is related to lung disease severity and thought to be caused by chronic inflammation. Increased iron levels in sputum are associated with P. aeruginosa infections.

Aim To establish the prevalence of ID and iron deficiency anemia (IDA) in children with CF and associations of ID with dietary iron intake, lung disease severity and Pseudomonas aeruginosa infection.

Methods Clinical charts of 54 children with CF aged 0 to 16 were reviewed. Follow-up varied from 1 to 14 years with 346 annual observations in total. Laboratory data (hemoglobin $(\mathrm{Hb})$, serum ferritin (SF)) and results of pulmonary function tests, sputum cultures and 3-day food records were collected.

Results 46 children $(85.2 \%)$ were iron deficient $(\mathrm{SF}<30 \mu \mathrm{g} / \mathrm{l})$ in at least one year and ID was present in 329 of 346 observations (95.1\%). IDA ( $\mathrm{SF}<30 \mu \mathrm{g} / \mathrm{l}$ and $\mathrm{Hb}>2 \mathrm{SD}$ below the mean of similarly aged children) was present in 8 observations $(2.4 \%)$ in 6 patients $(11.1 \%)$. Children with ID were younger (6.4 year versus 10.6 year, $p=0.00$ ) and had less pulmonary exacerbations ( $p=0.01$ ). ID was not associated with FEV1, Pseudomonas aeruginosa infection or dietary iron intake.

Conclusion ID is common in young children with CF and associated with less pulmonary exacerbations. We suggest that ID in these children is caused by rapid growth and accelerated erythropoies instead of disease severity or insufficient dietary iron intake.

\section{SERUM HEPCIDIN IN CHILDREN WITH BETA-THALASSEMIA}

doi:10.1136/archdischild-2012-302724.0772

${ }^{1} \mathrm{HM}$ Assem, ${ }^{2} \mathrm{OA}$ Sharaki, ${ }^{3} \mathrm{ME}$ El-Shennawi, ${ }^{3} \mathrm{HM}$ Gomaa. 'Pediatrics, Faculty of Medicine, Beirut Arab University, Beirut, Lebanon; ${ }^{2}$ Clinical Pathology; ${ }^{3}$ Pediatrics, Faculty of Medicine - Alexandria University, Alexandria, Egypt

Background Hepcidin, first described about 10 years ago, is a key iron - regulatory hormone. However, hepcidin measurement in a variety of human disease states are still lacking.

Aim To study serum level of hepcidin hormone in children with beta-thalassemia major (TM) and intermedia (TI)

Subjects and Methods The work was conducted on 50 children divided into 3 groups: 15 children with beta-thalassemia major, 10 children with beta-thalassemia intermedia, and 25 healthy children as a control group.

Thalassemic children included in the study were subjected to: Detailed history taking, clinical examination and measurement of serum hepcidin hormone level by (ELISA).

Results The mean serum hepcidin level was significantly higher in children with TM than in patients with TI and the controls. The ratio of serum hepcidin to serum ferritin in TI was significantly lower than those with TM. In addition, there was a significant positive relation between serum hepcidin and serum ferritin and also with serum iron.

Conclusions Hepcidin measurement may be useful as part of the diagnostic and prognostic evaluation of thalassemia as it may allow a more accurate assessment of the degree of iron overload and the maldistribution of iron.

In the future, it may be possible to use exogenous hepcidin to restore normal iron homeostasis in patients with thalassemia especially thalassemia intermedia.

\section{PLATELETS ANTIBODIES AND SERUM LEPTIN IN CHILDHOOD IMIMUNOTHROMBOCYTOPENIC PURPURA}

doi:10.1136/archdischild-2012-302724.0773

${ }^{1}$ HB Hamed, ${ }^{2}$ AM Zahran. 'Clinical Pathology, South Egypt Cancer Institute Assiut University; ${ }^{2}$ Clinical Pathology, Assiut University, Assiut, Egypt

Background and Aim Platelets antigens, Anti-platelets antibodies, serum leptin measurement may be important in defining the pathogenesis of thrombocytopenic states.

Methods In this study we measured the platelets CD41, CD61, CD62P, Platelets IgG, IgM by flowcytometry and serum leptin by ELISA of 20 children diagnosed as ITP and 20 normal children as control.

Results We observed That there were no significant difference in white blood cells count, hemoglobin concentration between ITP patients and controls. Platelets count was significantly decreased, and mean platelet volume (MPV) was significantly increased in patients than controls $\mathrm{P}=0.000$. The percentage of $\mathrm{CD} 41$ expressing platelets was significantly lower in ITP children compared to controls $(\mathrm{P}=0.001)$ but the percentage of $\mathrm{CD} 61$ expressing platelets was not significantly different between ITP patients and controls. Platelet activation marker CD62P was significantly expressed in patients than controls (0.000). Furthermore, the amount of CD62P per cell, represented by the MFI was significantly higher in patients than controls (0.000). The percentage of platelets associated IgM and IgC were was significantly increased in patients than controls $(\mathrm{P}=0.000)$ Also the MFI of IgM and IgG were significantly higher in patients than controls .Finally the concentration of serum leptin was increased in patients than controls $(\mathrm{P}=0.000)$ (table 2$)$. There was a negative correlation between The platelets count and Platelets IgG $(\mathrm{P}=0.000$ and $\mathrm{r}=-0.88)$.

Conclusion We concluded that The demonstration of antiplatelet antibodies (PAIgG, PAIgM) and decreased detection of platelet 